\title{
Quiz: ECG - Pacemaker
}

The following 12-lead electrocardiogram (ECG) tracing is provided from a 72 years old male patient implanted with a dual-chamber pacemaker (Medtronic Astra DR), when applying a magnet. He underwent three previous persistent atrial fibrillation and atrial tachycardia radiofrequency ablations, and an additional atrioventricular node ablation procedure (left ventricular ejection fraction $45 \%$ ). The patient does not report any cardiovascular symptom.

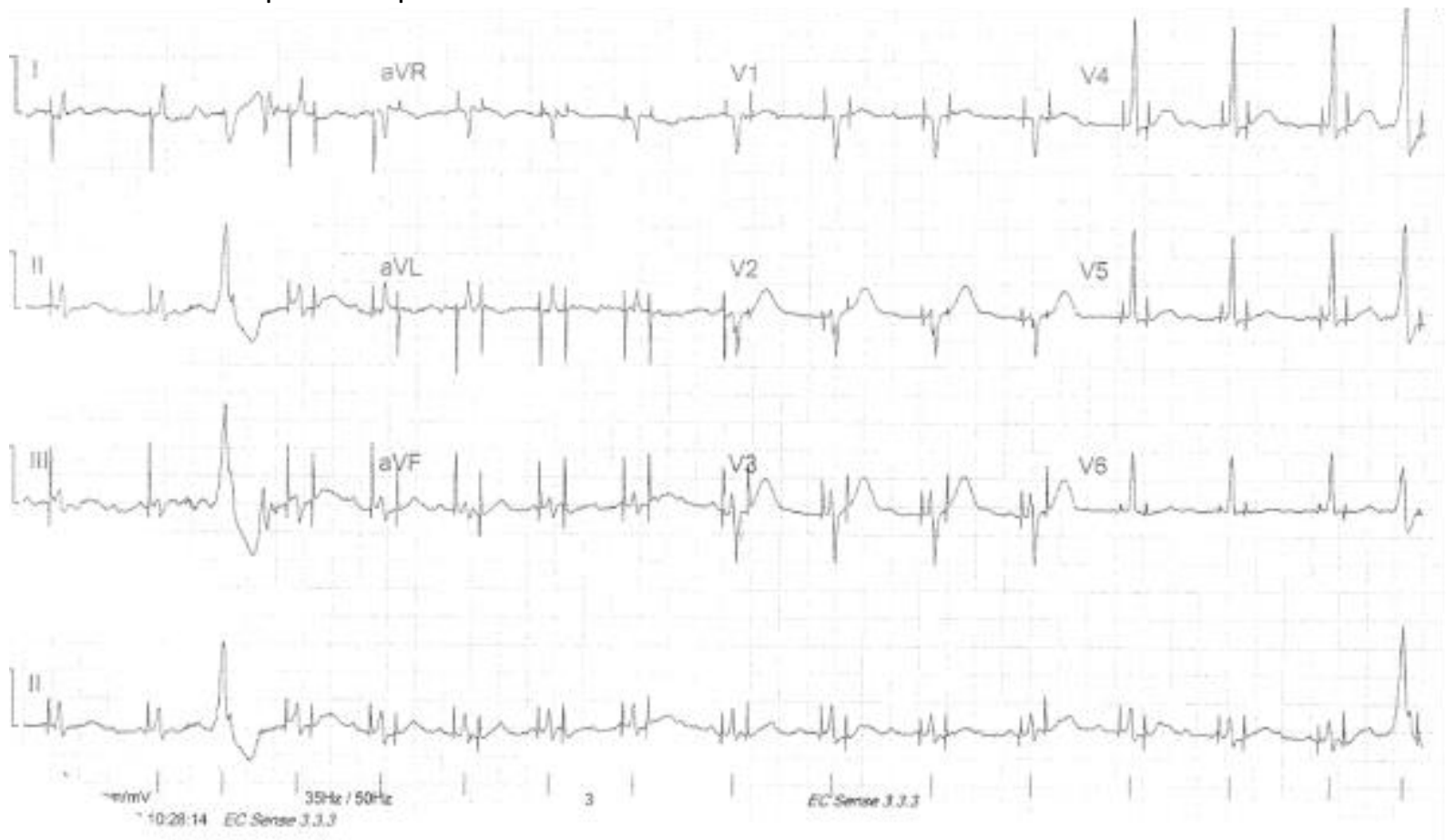

Figure 1. A 12-lead electrocardiogram recording

Which sentence of the following is correct?

1. The pacing leads have been connected to the incorrect port of the pacemaker.

2. There is a ventricular lead dysfunction because the second spike is not followed by any paced ventricular complex.

3. Fusion between native QRS and paced QRS is present as QRS complexes are narrow.

4. This tracing demonstrates normal magnet response to this pacing system.
5. The patient is in sinus rhythm

Sok-Sithikun BUN ${ }^{1}$, Abdelkarim Errahmouni ${ }^{2}$

${ }^{1}$ Pasteur University Hospital, Nice, France

${ }^{2}$ Dupuytren University Hospital, Limoges, France

Peer-review: Internal

Conflict of interest: None to declare

Authorship: S.S.B. and A.E. equally contributed to preparation of quiz

Acknowledgement and funding: None to declare 